\title{
Coal-rock interface recognition based on permutation entropy of LMD and supervised Kohonen neural network
}

\author{
Yong $\mathrm{Li}^{1}$, Gang Cheng ${ }^{1 * *}$, Xihui Chen ${ }^{2}$ and Chang Liu ${ }^{1}$ \\ ${ }^{1}$ School of Mechatronic Engineering, China University of Mining and Technology, Xuzhou 221116, China \\ ${ }^{2}$ College of Mechanical and Electrical Engineering, Hohai University, Changzhou 213022, China
}

\begin{abstract}
Owing to the difficulty in coal-rock interface recognition during the process of coal mining, the shearer is damaged at a high frequency. To avoid this problem, a method is proposed for coal-rock interface recognition based on permutation entropy calculated using the local mean decomposition (LMD) method and supervised Kohonen neural network (SKNN) by performing sound signal analysis. The complex and nonstationary sound signal is adaptively decomposed by LMD. Given that the decomposed product function (PF) components contain the main information of the features, permutation entropy (PE) is used to reflect the complexity and irregularity in each PF component and is defined as the input of the SKNN model. Finally, the optimal SKNN model is obtained by training the samples. The experimental results show that the comprehensive recognition rate of a coal-rock interface is up to $89 \%$. A coal-rock interface can be recognized effectively by sound signal analysis.
\end{abstract}

Keywords: Coal-rock recognition, local mean decomposition, permutation entropy, supervised Kohonen neural network, sound signal.

AUTOMATIC recognition technology for a coal-rock interface is the key technology in coal mining; it is of tremendous significance in the improvement of the service life of the associated equipment. It ensures the safety of workers and improves the efficiency of coal mining. Numerous methods such as the $\gamma$-ray, memory cutting, image and vibration detection methods have been proposed $^{1}$. However, there are a few limitations of these methods. The $\gamma$-ray method requires that the top and bottom plates be composed of radioactive elements; it can be used for only $20 \%$ of the coal mines in China ${ }^{2}$. With the image detection method, it is difficult to obtain the ideal original image owing to the effect of coal dust on the image acquisition device ${ }^{3}$. The vibration detection method utilizes the principle that the physical and mechanical properties of coal and rock are different, to achieve coal-rock interface recognition ${ }^{4}$. However, the vibration detection method is a contact signal acquisition

\footnotetext{
*For correspondence. (e-mail: chg@cumt.edu.cn)
}

method. The feature of a vibration signal is weakened by its transmission; this is not conducive for feature extraction. In contrast, a sound sensor adopts a non-contact signal acquisition method. It can drastically reduce the loss of the signal feature. The differences in the amplitudes, energies and frequencies of different signals can be determined by analysing the sound signal in the time and frequency domains.

The core of coal-rock interface recognition is feature extraction and state recognition. Considering the complex and non-stationary features of coal-rock sound signals, early scholars generally used the empirical mode decomposition (EMD) method to deal with the problem. However, there are issues regarding modal aliasing and endpoint leakage in the EMD process ${ }^{5}$. Smith ${ }^{6}$ proposed a new method of performing adaptive, non-stationary signal processing based on a previous study, called local mean decomposition (LMD). LMD decomposes a complex non-stationary signal adaptively into numerous product function (PF) components with instantaneous frequencies that have physical interpretations. Each PF component is multiplied by an envelope signal and a pure frequency modulation (FM) signal. By combining the instantaneous frequency and instantaneous amplitude of all the PF components, the complete time-frequency distribution of the original signal can be obtained. Compared with EMD, LMD has a good control over the number of iterations, and it improves the processing speed of the data. The average envelope function is obtained by using the moving average method during the process of decomposition. It can effectively suppress the endpoint effect ${ }^{7}$. The use of representative features such as energy, sample entropy, and kurtosis can effectively achieve the classification of the original signal ${ }^{8}$. Permutation entropy is a new method to study the irregular and nonlinear systems in nature. It can be used to describe a complex system quantitatively simplistically. Permutation entropy reflects the complexity in a one-dimensional time series, and has a high sensitivity for signal changes ${ }^{9}$.

The main function of state recognition is to identify the signal type by analysing the extracted signal features, and a neural network is widely used in this field. The Kohonen neural network (KNN) is one type of neural network. 
The KNN was proposed by Kohonen of the University of Helsinki, Finland ${ }^{10}$. It is a type of self-organizing competitive neural network and has a high operating speed. It can be used to realize an unsupervised classification of unknown date ${ }^{11,12}$. However, the classification results of the same data class correspond to different network neurons. To solve this problem, a supervised Kohonen neural network (SKNN) adds a supervised classification function to the $\mathrm{KNN}^{13}$. By adding an output layer after the competitive layer, the sound signals of coal and rocks can be distinguished directly. SKNN is a competitive neural network with simple network structure and it is suitable for dealing with classification problems with fewer samples and fewer types. However, it has some limitations in dealing with complex classification problems. In contrast, stacked auto encoder (SAE) based on auto encoder (AE) and deep belief networks (DBN) based on restricted Boltzmann machine (RBM) have significant advantages in dealing with multi-sample and multi-type classification $^{14,15}$. But when dealing with simple classification problems based on small samples, it is prone to overfitting. Moreover, compared to SKNN, complex network structure takes more time to train. This article is aimed at classifying cutting types of shearers, which are divided into two types: coal and rock. Therefore, SKNN is used to realize data classification.

The remainder of this article is organized as follows: in the next section, the mathematical model for the coalrock interface recognition based on the permutation entropy calculated using the LMD and SKNN is established. Then the method for identifying the original sound signal used in coal-rock interface recognition and the relative parameters of the sensor are introduced in detail. The decomposition of the sound signal into several PF components and calculation of the permutation entropy of each PF component is then discussed. A representative component is selected as the input for the SKNN. By using a sample signal to train the SKNN, the optimal neural network can be obtained. The coal-rock cutting sound signal can be recognized by the trained neural network. In the last section, some conclusions are drawn.

\section{Mathematical model of coal-rock interface recognition}

The sound signal produced by the coal-rock cutting of an underground coal mine has complex and non-stationary features. It is difficult to extract the signal features from the time domain signal directly. By using the method of LMD to process the sound signal, the complete time-frequency distribution of the original signal can be obtained ${ }^{16}$.

\section{Local mean decomposition}

LMD adaptively decomposes a complex non-stationary signal into numerous PF components. Each PF compo- nent is multiplied by an envelope signal and a pure FM signal $^{17}$. The process of LMD is outlined as follows.

Step 1: All local extreme points $n_{i}$ of signal $x(t)$ are located. Mean value $m_{i}$ and envelope estimate $a_{i}$ of an adjacent local extreme are calculated as follows

$$
\begin{aligned}
& m_{i}=\frac{n_{i}+n_{i+1}}{2}, \\
& a_{i}=\frac{\left|n_{i}-n_{i+1}\right|}{2} .
\end{aligned}
$$

All adjacent mean values $m_{i}$ and adjacent envelope estimates $a_{i}$ are connected by straight lines by the method of moving average. Local mean function $m_{11}(t)$ and envelope estimation function $a_{11}(t)$ are obtained.

Step 2: Mean value function $m_{11}(t)$ separated from original signal $x(t)$ is demodulated to obtain FM signal $s_{11}(t)$ as follows

$$
s_{11}(t)=\frac{x(t)-m_{11}(t)}{a_{11}(t)} .
$$

Step 3: FM signal $s_{11}(t)$ is defined as a new signal and its envelope estimation function $a_{12}(t)$ is calculated by Step 1. If $a_{12}(t)$ is not equal to $1, \mathrm{FM}$ signal $s_{11}(t)$ is not a pure FM signal and requires further processing. Steps 1 and 2 are repeated until envelope estimate function $a_{1(n+1)}(t)$ of FM signal $s_{1 n}(t)$ becomes equal to one. In practical applications, the iteration terminates when $a_{1(n+1)}(t)$ is approximately equal to one ${ }^{18}$

$$
\lim _{x \rightarrow \infty} a_{1(n+1)}(t) \approx 1 \text {. }
$$

Step 4: The envelope signal is multiplied by all the envelope estimation functions generated in the iterative process

$$
a_{1}(t)=a_{11}(t) a_{12}(t) \ldots a_{1 n}(t)=\prod_{q=1}^{n} a_{1 q}(t) .
$$

Step 5: The first PF component is obtained as follows

$$
\mathrm{PF}_{1}(t)=a_{1}(t) s_{1 n}(t)
$$

It is a single component that contains the highest frequency component of the original signal. The instantaneous amplitude is envelope signal $a_{1}(t)$. The instantaneous frequency can be obtained as follows

$$
f_{1}(t)=\frac{\pi}{2} \frac{d\left[\arccos \left(s_{1 n}(t)\right)\right]}{d_{t}} .
$$


Step 6: Residual signal $u_{1}(t)$ is obtained by extracting the first $\mathrm{PF}$ component from original signal $x(t) . u_{1}(t)$ is defined as a new signal, and the above-mentioned steps are repeated $k$ times until $u_{k}(t)$ becomes a monotonic function. The original signal can be expressed as follows

$$
x(t)=\sum_{p=1}^{k} \mathrm{PF}_{p}(t)+u_{k}(t) .
$$

\section{Permutation entropy}

Various PF components with physical characteristics can be obtained by the LMD that contains numerous instantaneous amplitude frequency features. Using the permutation entropy to extract the features of each layer, the amplitude frequency features of the signal can be described quantitatively ${ }^{19}$, as follows.

Assuming that $\mathrm{PF}=\{x(i), i=1,2,3, \ldots, N\}$, the following set of matrix $X$ can be obtained

$$
X=\left\{\begin{array}{llll}
x(1) & x(1+\tau) & \ldots & x(1+(d-1) \tau) \\
x(2) & x(2+\tau) & \ldots & x(2+(d-1) \tau) \\
x(j) & x(j+\tau) & \ldots & x(j+(d-1) \tau) \\
\ldots & \ldots & \ldots & \ldots \\
x(k) & x(k+\tau) & \ldots & x(k+(d-1) \tau)
\end{array}\right\}
$$

where $\tau$ is the delay time, $d$ the embedding dimension, $k$ the number of reconstructed components and $x(j)$ is line $j$ component of the reconstruction matrix.

Each row in matrix $X$ can be considered as a separate reconstruction component, and the number of reconstructed components is $k=N-(d-1) \tau$. Each row in the reconstruction matrix $X$ is re-arranged in ascending order as follows

$$
\begin{aligned}
& x\left(i+\left(j_{1}-1\right) \tau\right) \leq x\left(i+\left(j_{2}-1\right) \tau\right) \\
& \leq \ldots \leq x\left(i+\left(j_{d}-1\right) \tau\right),
\end{aligned}
$$

where $j_{1}, j_{2}, \ldots, j_{d}$ represent the indices of the columns in each element of the reconstruction component.

If there are equal values in the reconstruction component as given below, then $j_{p}$ and $j_{q}$ are sorted according to the size of the value

$$
y\left(i+\left(j_{p}-1\right) \tau\right)=y\left(i+\left(j_{q}-1\right) \tau\right)
$$

The columns of an element of the reconstruction component are sorted as follows when $j_{p}<j_{q}$

$$
y\left(i+\left(j_{p}-1\right) \tau\right) \leq y\left(i+\left(j_{q}-1\right) \tau\right)
$$

Therefore, a set of symbolic sequences can be obtained from each line of matrix $Y$ of any time series, as follows

$$
S(l)=\left\{j_{1}, j_{2}, \ldots, j_{d}\right\}
$$

where $l=1,2, \ldots, n$. There are $m$ ! types of different symbolic sequences in an $m$ dimensional space and $n<m$ !, and symbol sequence $S(l)$ is one of them. The probability of each symbol sequence $P_{k}$ is calculated, and the permutation entropy of time series $x(i)$ can be defined in terms of the information entropy as follows

$$
H_{p}(d)=-\sum_{j=1}^{n} P_{j} \ln \left(P_{j}\right)
$$

$H_{p}(d)$ attains the maximum value $\ln (d !)$ when $P_{j}=1 / d$ !. $H_{p}(d)$ is usually normalized as follows for convenience

$$
0 \leq H_{p}=\frac{H_{p}}{\ln (d !)} \leq 1 .
$$

The value of $H_{p}$ represents the random degree of a time series $\{x(i), i=1,2, \ldots, N\}$. A small value of $H_{p}$ implies more regularity in the time series. In contrast, the time series is nearly random. A change in $H_{p}$ reflects the small details of the time series.

\section{Supervised Kohonen neural network}

By using permutation entropy, the features of the original signal can be represented as values. The SKNN realizes the recognition and classification of signal types by analysing the array of such values ${ }^{20}$. It is composed of an input layer, a competitive layer, and an output layer. Its structure is shown in Figure 1. The training steps in the SKNN are shown as follows.

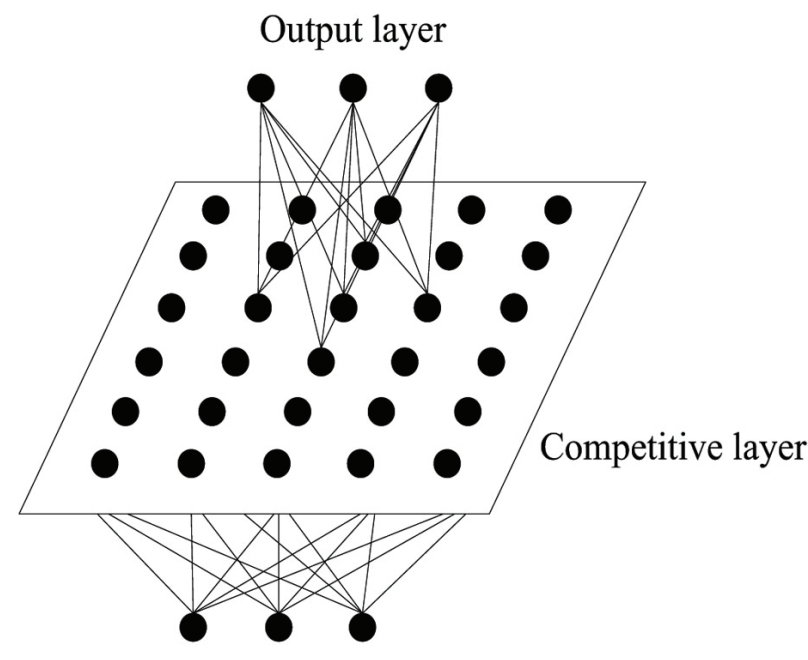

Input layer

Figure 1. Structure of the SKNN. 


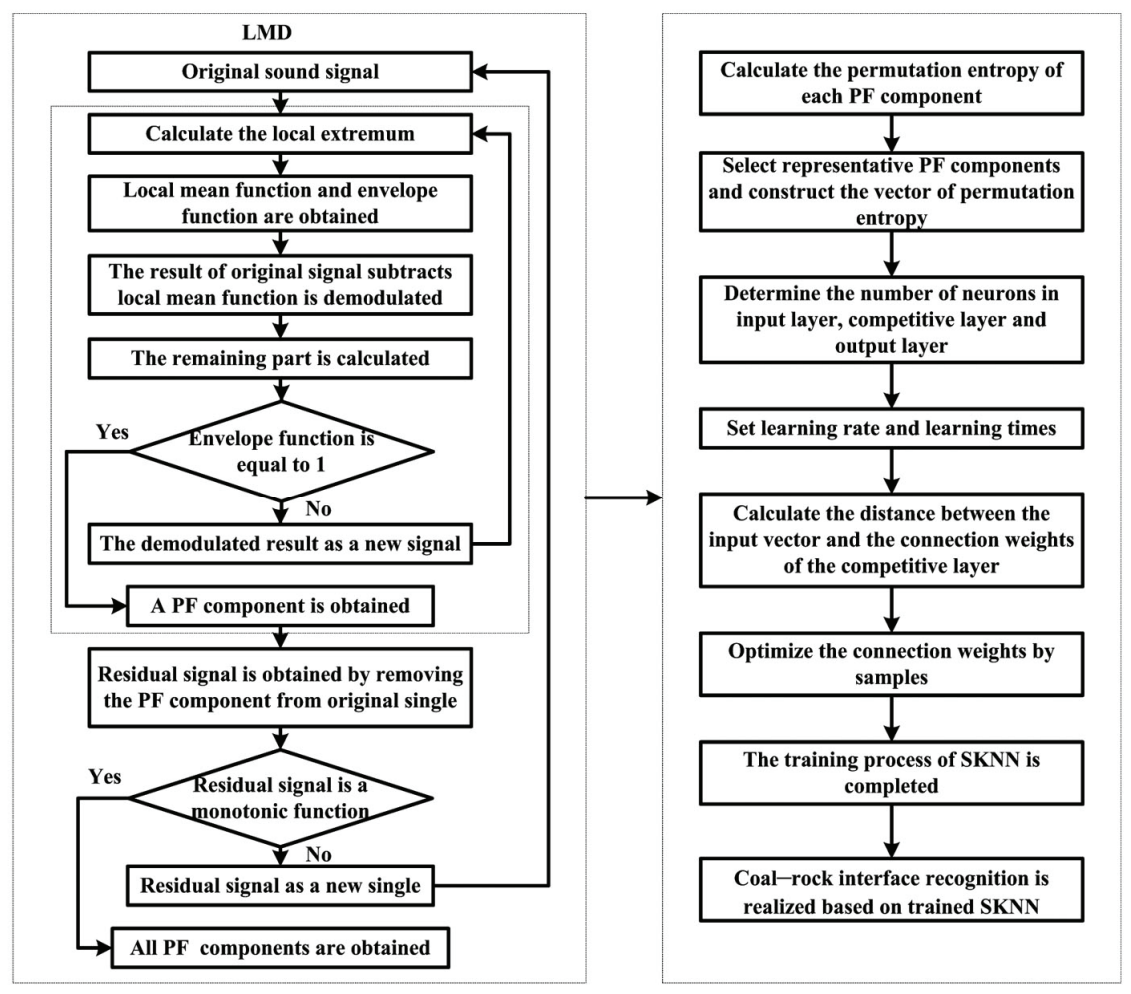

Figure 2. Flowchart of experimental analysis.

Step 1: Initializing the network. The structure of a neural network is determined according to number of input layer neurons $m$ and number of output layer neurons $g$. The number of iterations is set to $T$, and the connection weights of the input layer and output layer neurons are randomly initialized to arrays $w_{i j}$ and $w_{j k}$ that are between 0 and 1 . The learning rates and field radius are set to $\eta_{1}$, $\eta_{2}$, and $r$, where $i=1,2, \ldots, m ; j=1,2, \ldots, n$; and $k=1$, $2, \ldots, g$.

Step 2: Learning rates $\eta_{1}$ and $\eta_{2}$ are updated as follows

$$
\begin{aligned}
& \eta_{1}=\eta_{1}-\frac{i}{T} \eta_{1}, \\
& \eta_{2}=\eta_{2}-\frac{i}{T} \eta_{2} .
\end{aligned}
$$

Step 3: Distance $d$ between input vector $X=\left(x_{1}, x_{2}, \ldots, x_{m}\right)$ and value $w_{i j}$ of the competitive layer neuron is calculated. Neuron $c$ of the competitive layer that is mostly close to input vector $X$ is chosen as the optimal winning neuron. All neurons $N_{C}(t)$ in the domain of radius $r$ of neuron $c$ are selected as follows

$$
d_{c}=\min \left(\sqrt{\sum_{i=1}^{m}\left(x_{i}-w_{i j}\right)^{2}}\right) \quad i=1,2, \ldots, m, j=1,2, \ldots, n
$$

$$
N_{c}(t)=\left(t \mid \operatorname{find}\left(\operatorname{norm}\left(\operatorname{pos}_{t}, \operatorname{pos}_{c}\right)<r\right)\right) \quad t=1,2, \ldots, n,
$$

where $\operatorname{pos}_{c}$ and $\operatorname{pos}_{t}$ are the positions of neurons $c$ and $t$ respectively, norm is the Euclidean distance between two neurons, and $r$ is the radius of the field.

Step 4: The distances between selected $w_{j k}$ and the output layer neurons are calculated, and the output layer neuron with minimum distance is selected to determine the type of data

$$
d_{a}=\min \left(\sqrt{\sum_{j=1}^{n}\left(y_{j}-w_{j k}\right)^{2}}\right) \quad j=1,2, \ldots, n, g=1,2, \ldots, k
$$

Step 5: Connection weights $w_{i j}$ and $w_{j k}$ are decreased linearly with the times of learning. There are adjusted as follows

$$
\begin{aligned}
& w_{i j}=w_{i j}+\eta_{1}\left(X_{i}-w_{i j}\right), \\
& w_{j k}=w_{j k}+\eta_{2}\left(Y_{j}-w_{j k}\right) .
\end{aligned}
$$

\section{Flowchart of the algorithm}

The experimental analysis flowchart is shown in Figure 2. 


\section{Test equipment and data acquisition}

The experiment was conducted in the Baodian coal mine of the Yanzhou Mining Group, which is shown in Figure 3.

To control the effect of external factors and ensure the reliability of the experiment, the shearer is set at the same speed to cut the coal and rock. The sound signal of the rocker arm of MG900/2400-WD electric traction shearer is collected by the sound sensor that is shown in Figure 4. The sampling frequency is set as $44 \mathrm{kHz}$.

The main technical specifications and features of the sound sensor are shown in Table 1.

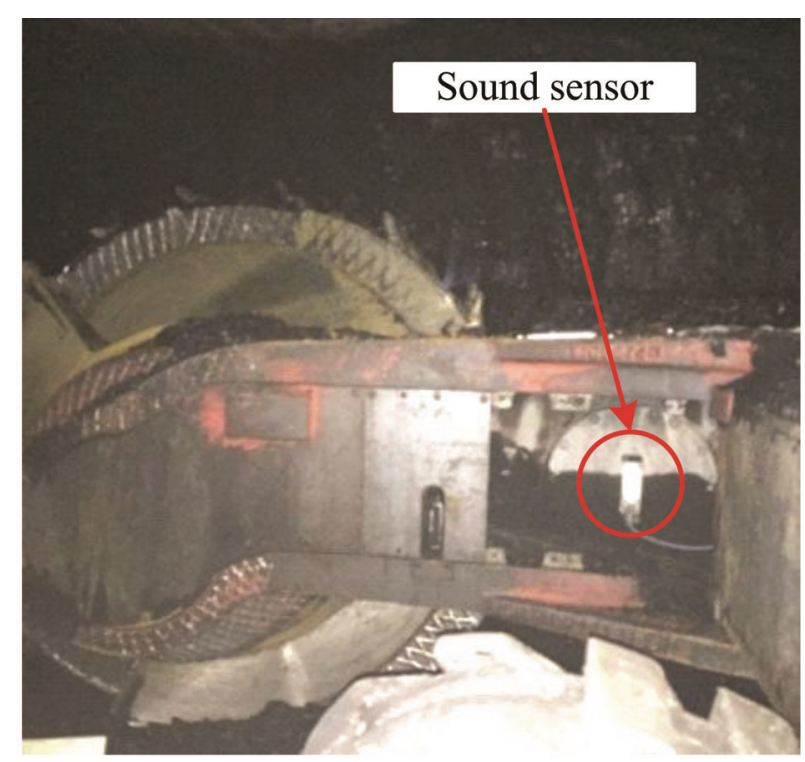

Figure 3. Site of signal acquisition.

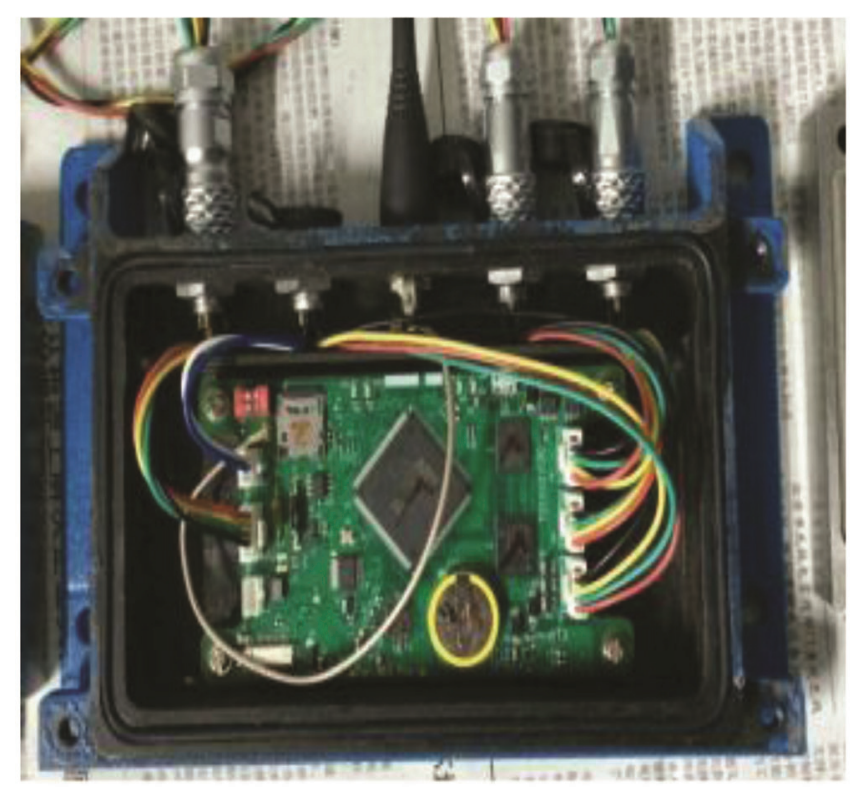

Figure 4. Sound signal processing device.

\section{Experimental analysis}

The accuracy and effectiveness of the proposed coal-rock interface recognition method are verified by analysing two types of sound signals. The two types of sound signals that are collected are shown in Figure 5.

It can be seen that there is no obvious difference between the two types of signals in terms of the amplitude. The relevant part of the sound signal not only distributes in the time domain, but also exists in the form of shock in the non-stationary signal. The analysis based on the time domain only reflects the fluctuation of the sound signal and cannot reproduce the entire features of the measured data. It is difficult to classify the sound signal of the coal-rock cutting. LMD is used for processing to extract the features of the original signal.

100 coal cutting samples and 100 rock cutting samples are intercepted from the original signal. Each data contains 8800 data points and is processed by LMD. Figure 6 displays a comparison of the sound signals of the coalrock cutting.

It can be seen from Figure 6 that LMD decomposes the original signal into six PF components with different frequencies and one residual component $u$. By comparing the same component of the two signals, we can see that the frequencies of the two signals are similar, but there are some differences in amplitude fluctuation. To extract

Table 1. The main technical specifications and features of the sound sensor

\begin{tabular}{ll}
\hline Technical specifications & \multicolumn{1}{c}{ Value } \\
\hline Range of acceleration detection & $\pm 50 \mathrm{~g}$ \\
Bandwidth of signal detection & $10 \mathrm{~Hz}-5000 \mathrm{~Hz}$ \\
Transform resolution of ADC & $21 \mathrm{bit}$ \\
Conversion rate of ADC & $12 \mathrm{kHz}, 24 \mathrm{kHz}, 48 \mathrm{kHz}$ \\
Range of sound detection & $40-110 \mathrm{~dB}$ \\
\hline
\end{tabular}
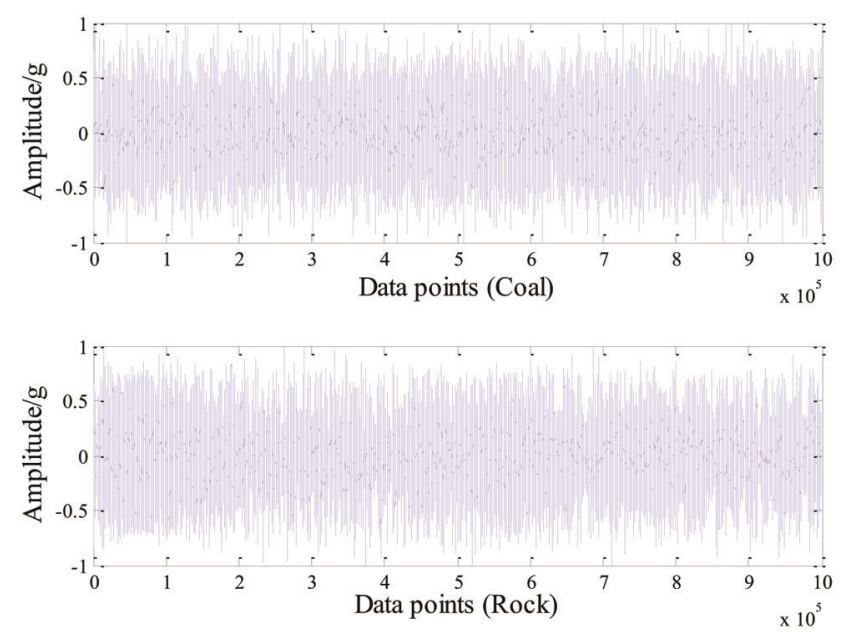

Figure 5. Original sound signals.

CURRENT SCIENCE, VOL. 116, NO. 1, 10 JANUARY 2019 
the feature parameters of the signal, permutation entropy is used to process each component to reflect the complexity of the one-dimensional time series with a high sensitivity to changes in the signal ${ }^{21}$.

In view of the scope of this study, we randomly selected ten groups of samples from the two types of signals for the analysis. The permutation entropy value of each PF component and variation tendency of the mean are shown in Figure 7.

It can be seen from Figure 7 that the permutation entropy of each PF component of the rock cutting sound signal is generally larger than that of the coal cutting sound signal. However, the permutation entropy of the two signals still exists as a cross-distribution, particularly in each layer. This phenomenon is particularly significant

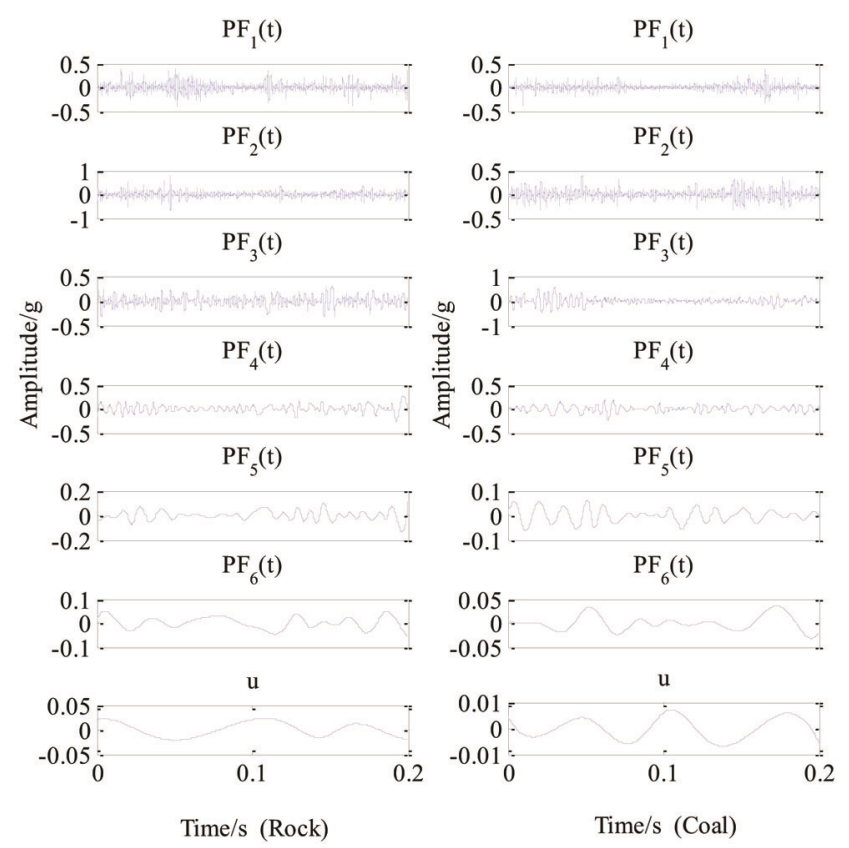

Figure 6. Results of LMD of the sound signals.

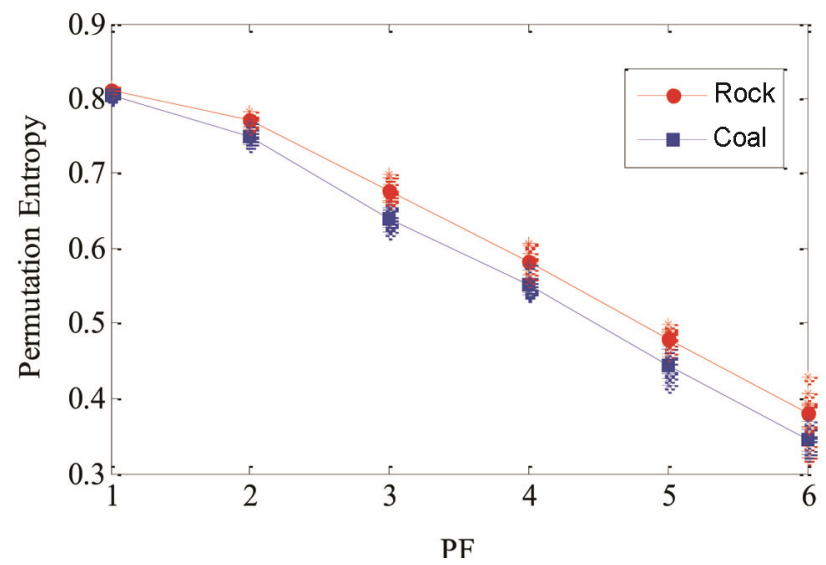

Figure 7. Permutation entropy value of each PF component and the variation tendency of the mean. in the first and last layers, and makes it impossible to reflect the difference between the signals of coal and rock. To realize the classification of signals, we chose the permutation entropies of the middle four layers as the feature parameter. The phenomenon of cross-distribution in the middle layers is minor, and the permutation entropies can satisfactorily reflect the features of the coal-rock sound signals. To eliminate the recognition error caused by the cross-distribution, the permutation entropies of the four middle layers are used as the input feature vector of the neural network.

The recognition of the coal-rock interface is realized by using the SKNN. The sound signal must be accurately divided into two types; therefore, the output layer of the SKNN has two neurons. The output of the sound signal is represented by [ $\left.\begin{array}{ll}0 & 1\end{array}\right]$ and [ $\left[\begin{array}{ll}1 & 0\end{array}\right]$, where [ $\left[\begin{array}{ll}0 & 1\end{array}\right]$ represents the

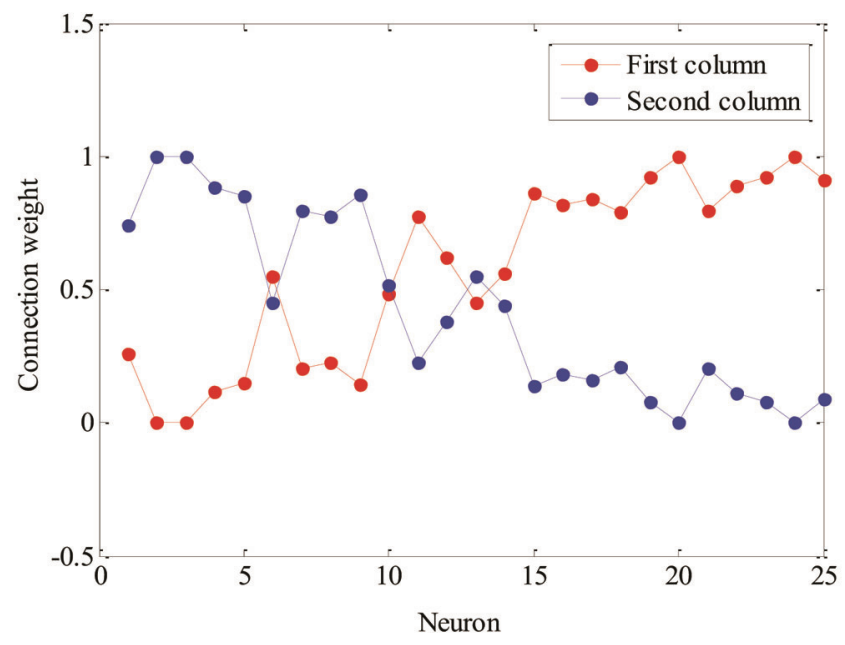

Figure 8. Connection weights of output layer $w_{j k}$.

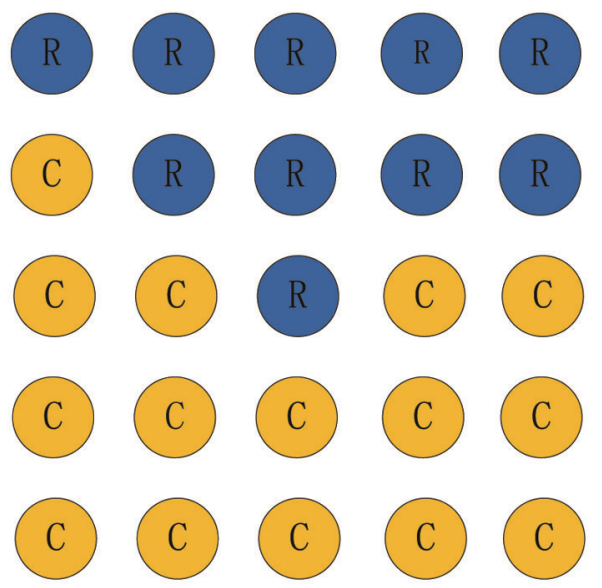

$\mathrm{R}$ represents the winning neurons of rock signal $\mathrm{C}$ represents the winning neurons of coal signal

Figure 9. Classification results of the competitive layer neurons. 
Table 2. Recognition rates of the different types of signals

\begin{tabular}{lcl}
\hline Method & Recognition rate (\%) & \multicolumn{1}{c}{ Remarks } \\
\hline LMD-PE-SKNN & 89 & Half samples for training, half samples for testing \\
SAE & 58 & Half samples for training, half samples for testing \\
& 97 & All samples for training and testing \\
DBN & 56 & Half samples for training, half samples for testing \\
& 84 & All samples for training and testing \\
\hline
\end{tabular}

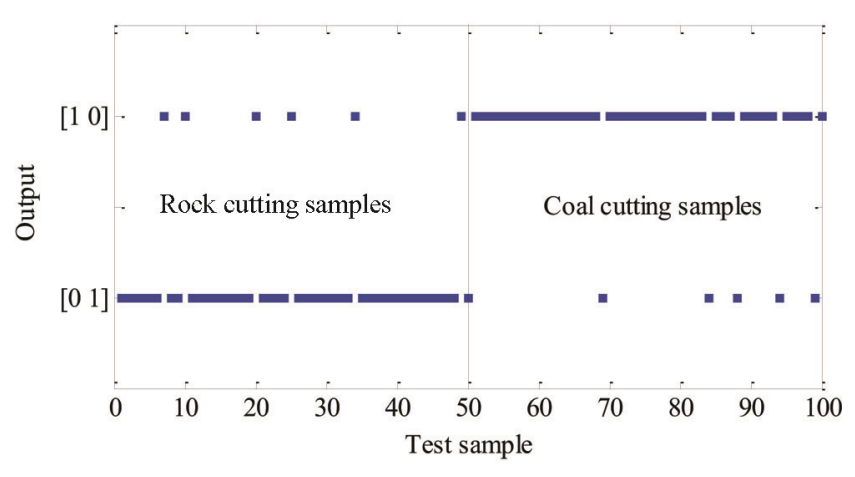

Figure 10. Recognition result of the validation samples.

sound signal of the rock and [1 0 ] represents that of coal. The size of the competitive layer is mainly related to the number of data categories. When there are fewer neurons and more categories, the centres of each category are squeezed and shift close to each other. Each type cannot be well represented. In contrast, it takes a long time for the network to converge when the number of neurons is large $\mathrm{e}^{22}$. Considering the number of input and output neurons, $5 * 5$ distribution mode is chosen as the competitive layer in this study, and an SKNN with the 4-25-2 structure is constructed.

The number of iterations of the network training $t$ is set to 1000 , and the initial learning rate of connection weights $w_{i j}$ and $w_{j k}$ are set to 1 . The radius of the field is set to 2, according to the size of the competitive layer. Fifty per cent of the data of the sound signals processed by the PF permutation entropy are randomly selected as training samples for the random mixing and the remaining $50 \%$ are used as validation samples. The neural network is trained to obtain the optimal connection weights by reducing the learning rate. Trained weight $w_{j k}$ is shown in Figure 8.

The classification results of the competitive layer can be obtained by calculating the Euclidean distance between the connection weights and neurons of output layer which are shown in Figure 9.

By calculating the distance between the sample and each neuron in the competitive layer and searching for the superior neuron, the type of data can be determined. The recognition result of the sample is shown in Figure 10. The first 50 groups are the signals of rock cutting, and the latter 50 groups are the signals of coal cutting.
As can be seen from Figure 10, there are six errors in first 50 rock cutting samples and five recognition errors in the latter 50 coal cutting samples. The coal-rock interface can be recognized by analysing the sound signals and the comprehensive recognition rate of the coal-rock interface is up to $89 \%$.

By contrast, SAE and DBN are used for comparison. In order to prove the effectiveness of these two algorithms and the limitations in dealing with small samples, two experimental schemes are adopted in this paper. In one scheme, 200 samples are used as training samples and test samples simultaneously. In the second scheme, 200 samples are divided into two groups on average, 100 samples are training samples, and 100 samples are test samples. The recognition rate of the three methods in the experiment are shown in Table 2.

It can be seen from Table 2 that the coal-rock interface can be recognized by analysing the sound signals of the coal-rock cutting, and the comprehensive recognition rate of the coal-rock interface is up to $89 \%$. When all the samples are used for training and testing at the same time, the recognition rate of SAE and DBN can reach $97 \%$ and $84 \%$. This shows that these two methods can effectively mine the internal characteristics of data, and effectively establish the intrinsic link between original signals and category labels. However, when the test samples and training samples are inconsistent, the recognition rate dropped sharply. This shows that these two methods have problems such as over fitting in dealing with the classification problem of small samples, and cannot achieve efficient classification. A large number of samples are necessary for improving the rate of network recognition. Therefore, the coal rock recognition algorithm based on LMD, PE and SKNN which is proposed in this paper has obvious advantages in dealing with small sample data.

\section{Conclusion}

In the process of coal mining, it is important to recognize the coal-rock interface and adjust the mining plan to reduce the rocker arm failure and ensure the efficiency of coal mining. In this study, a method for coal-rock interface recognition based on the permutation entropy calculated using the LMD and SKNN is presented. LMD is used to decompose non-linear and non-stationary sound signals, and the permutation entropy is used to describe 
the characteristics of the signals quantitatively. Finally, the SKNN is used to classify the signals. Based on the analysis of the cutting signals of the coal-rock interface, the following conclusions are drawn.

As a non-contact signal, the sound signal of a coalrock cutting can effectively avoid problems existing in the acquisition process of other types of signals. It can better preserve the signal characteristics. Based on the algorithm for the permutation entropy calculated using the LMD and SKNN, the original signal can be decomposed efficiently, and the higher order features of the signals can be extracted to realize automatic signal classification. Using the proposed method, the recognition rate of the coal-rock interface reached $89 \%$, indicating that the proposed method can be used to realize the recognition of a coal-rock interface.

1. Ren, F., Yang, Z. J. and Xiong, S. B., Study on the coal-rock interface recognition method based on multi-sensor data fusion technique. Chin. J. Mech. Eng., 2003, 16(3), 321-324 (in Chinese).

2. Asfahani, J. and Borsaru, M., Low-activity spectrometric gammaray logging technique for delineation of coal-rock interfaces in dry blast holes. Appl. Radiat. Isot., 2007, 65(6), 748-755.

3. Sun, J. P., Study on identified method of coal and rock interface based on image recognition. Coal. Sci. Technol., 2011, 39(9), 7779 (in Chinese).

4. Wang, B. P., Wang, Z. C. and Zhang, W. Z., The method of coalrock interface recognition based on EMD and neural network. J. Vibrat. Measure. Diagnosis, 2012, 32(4), 586-590 (in Chinese).

5. Zhang, J., Ou, J. P. and Zhan, R. H., Automatic target recognition of moving target based on empirical mode decomposition and genetic algorithm support vector machine. J. Cent. South. Univ. Technol., 2015, 22(4), 1389-1396.

6. Han, M. H. and Pan, J. L., A fault diagnosis method combined with LMD, sample entropy and energy ratio for rolle. Measurement, 2015, 75(11), 7-19.

7. Kidar, T., Thomas, M., Guibault, R. and Badaoui, M., Comparison between the efficiency of LMD and EMD algorithms for early detection of gear defects. Mech. Ind., 2013, 14(2), 121-127.

8. Chen, X. H., Cheng, G. and Li, H. Y., Fault identification method for planetary gear based on DT-CWT threshold denoising and LE. J. Mech. Sci. Technol., 2017, 31(3), 1035-1047.

9. Yi, C. C., Lv, Y., Ge, M., Xiao, H. and Yu, X., Tensor singular spectrum decomposition algorithm based on permutation entropy for rolling bearing fault diagnosis. Entropy, 2017, 19(4), 139

10. Kohonen, T., Self-organization and associative memory: 3rd edition. Appl. Optics, 1989, 8(1), 3406-3409.
11. Cheng, G., Cheng, Y. L., Shen, L. H., Qiu, J. B. and Zhang, S., Gear fault identification based on Hilbert-Huang transform and SOM neural network. Measurement, 2013, 46(3), 1137-1146.

12. Galhardo, C. E. C. and Rocha, W. F. D., Exploratory analysis of biodiesel diesel blends by Kohonen neural networks and infrared spectroscopy. Anal. Methods, 2015, 7(8), 3512-3520.

13. Ghobadi, M. Z. and Kompany, Z. M., Application of supervised Kohonen map and counter propagation neural network for classification of nucleic acid structures based on their circular dichroism spectra. Spectrochim. Acta. A, 2014, 132(11), 345-354.

14. Zabalza, J., Ren, J. C. and Zheng, J. B., Novel segmented stacked autoencoder for effective dimensionality reduction and feature extraction in hyperspectral imaging. Neurocomputing, 2016, 185, $1-10$.

15. Liu, Y., Fan, Y. and Chen, J. H., Flame images for oxygen content prediction of combustion systems using DBN. Energy Fuels, 2017, 31(8), 8776-8783.

16. Goyal, D. and Pabla, B. S., The vibration monitoring methods and signal processing techniques for structural health monitoring: a review. Arch. Comput. Method. E, 2016, 23(4), 585-594.

17. Chen, X. H., Cheng, G., Li, H. Y. and Zhang, M., Diagnosing planetary gear faults using the fuzzy entropy of LMD and ANFIS. J. Mech. Sci. Technol., 2016, 30(6), 2453-2462.

18. Zhang, Y., Qin, Y. and Xing, Z. Y., Roller bearing safety region estimation and state identification based on LMD-PCA-LSSVM. Measurement, 2013, 46(3), 1315-1324.

19. Wei, Y., Xu, M. Q. and Li, Y. B., Gearbox fault diagnosis based on local mean decomposition, permutation entropy and extreme learning machine. J. Vibroeng., 2016, 18(3), 1459-1473.

20. De, A., Chakraborty, K. and Chakrabarti, A., Classification of power system voltage stability conditions using Kohonen's selforganising feature map and learning vector quantisation. Eur. T. Electr. Power, 2012, 22(3), 412-420.

21. Zhang, X. Y., Liang, Y. T., Zhou, J. Z. and Zang, Y., A novel bearing fault diagnosis model integrated permutation entropy, ensemble empirical mode decomposition and optimized SVM. Measurement, 2015, 69(7), 164-179.

22. Zhou, B. H., Li, X. and Fung, R. Y. K., Dynamic scheduling of photolithography process based on Kohonen neural network. J. Intell. Manuf., 2015, 26(1), 73-85.

ACKNOWLEDGEMENTS. This work was supported by a project funded by the Priority Academic Program Development of Jiangsu Higher Education Institutions and Fundamental Research Funds for the Central Universities (Grant number 2015YC02). This support is gratefully acknowledged.

Received 17 November 2017; revised accepted 20 September 2018

doi: $10.18520 / \mathrm{cs} / \mathrm{v} 116 / \mathrm{i} 1 / 96-103$ 